\title{
An adjustable Cosmological Constant
}

\author{
Jihn E. Kim
}

Department of Physics and Astronomy and Center for Theoretical Physics, Seoul National University, Seoul 151-747, Korea

DOI: http://dx.doi.org/10.3204/DESY-PROC-2010-03/kim_jihn

After showing the axion couplings, I review the Hawking type solution of the cosmological constant problem, present a (probably) correct way to calculate the probability amplitude, and show that the Kim-Kyae-Lee self-tuning model allows a finite range of parameters for the $\bar{\Lambda}=0$ to have a singularly large probability, approached from the AdS side.

\section{Axion couplings}

Since this is an axion conference, I present first the axion couplings with the current status and then discuss on the cosmological constant (CC). The axion couplings are given in [1] for the KSVZ [2] and DFSZ axions [3]. We show the current cosmological and astrophysical bounds in Fig. 1. Theortical expectations are also shown. In particular, we insert one line [4] from a superstring model calculation from a $Z_{12-I}$ compactification [5]. This is a calculation from a theory with an ultra violet completion with all fermions of the full theory is taken into account.
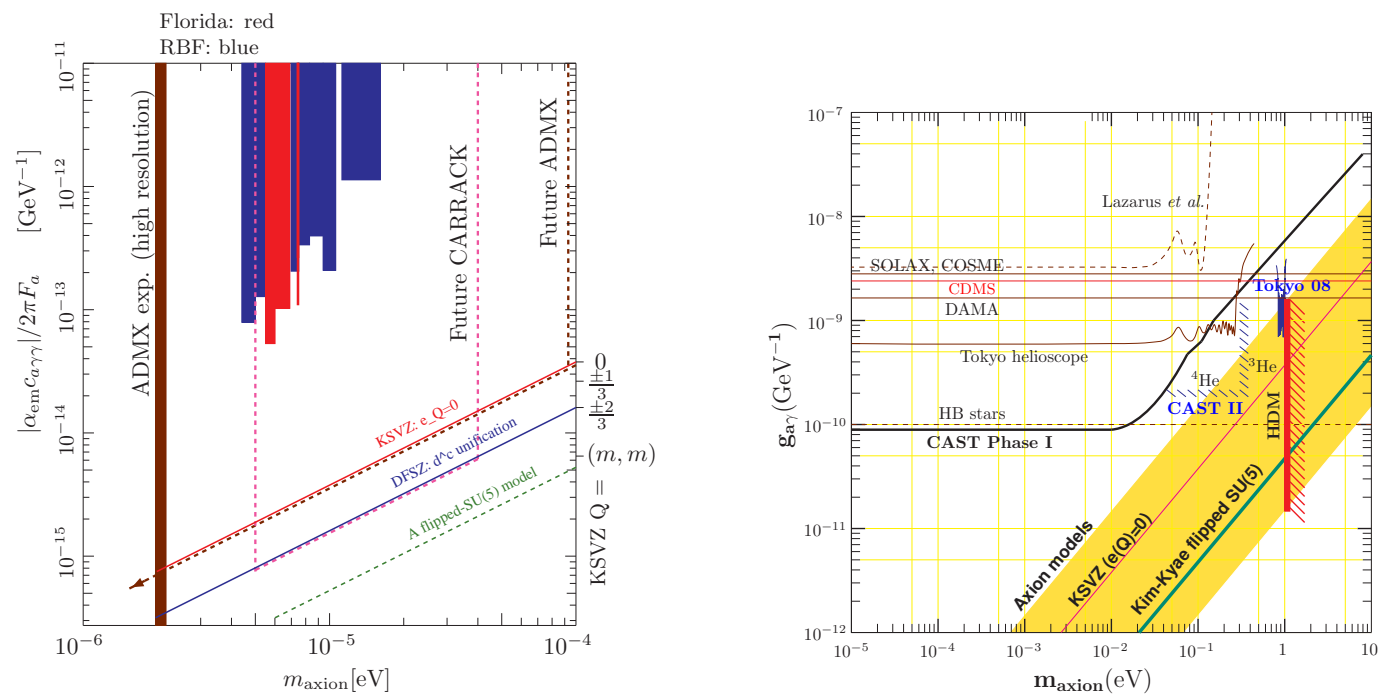

Figure 1: (a) The cosmological bounds and axion models, and (b) the astrophysical bounds. 


\section{Cosmological constants}

Now, I present the idea on the solution of the CC problem in models with extra dimensions published in $[6,7]$.

Recently, Nicoli commented (cited in [7]) that the Einstein equation is inconsistent from the outset because the left-hand side (LHS) and the right-hand side (RHS) of the Einstein equation $R_{\mu \nu}-\frac{1}{2} R g_{\mu \nu}=8 \pi G_{N} T_{\mu \nu}$ are logically different. The LHS is exactly determined by the continuous space-time geometry, and the RHS is contributed by particle quanta which have roots in the probabilistic interpretation of quantum mechanics. So, any discussion in the Planck era is speculative and not yet well formulated, and the present CC solutions are speculative. But they are welcome if it is not contradicted outrageously from the present perspective on the fundamental physics. In this spirit I present an idea with a specific action toward a $\mathrm{CC}$ solution.

One question on the determination of the $\mathrm{CC}$ is at which energy scale and temperature the $\mathrm{CC}$ is required to vanish. Out of the mass hierarchy scales in particle physics, from $M_{P}=2.44 \times 10^{18}$ $\mathrm{GeV}$ down to $T \approx 10^{-4} \mathrm{eV}$, the scale where the $\mathrm{CC}$ is determined must be known. Note that there are two other issues related to the CC constant. After the first inflationary scenario with the $R$ and $R^{2}$ terms [8], the inflationary paradigm has been formulated with a scalar field called the inflaton [9]. Presumably, the beginning of inflation with the inflaton starts at a temperature when the vacuum energy was much above the electroweak scale [9]. The vanishing CC solution at the zero temperature must be in harmony with this inflation of the early Universe. Another $\mathrm{CC}$ related issue is the current acceleration of the Universe which is known as the dark energy (DE) problem [10]. The current DE is not exactly zero but of order $(0.002 \mathrm{eV})^{4}$. The vanishing $\mathrm{CC}$ solution must be in harmony with this tiny $\mathrm{DE}$ also. If there exists a $\mathrm{CC}$ solution, it is better to address these other problems.

When we consider quantum mechanics, we talk in terms of the probability amplitude: The initial state $|I\rangle$ transforming to a final state $|F\rangle$. In this spirit, Baum and Hawking [11] considered the Euclidian action, only with the Ricci scalar $R$ and the CC term $\Lambda$. If the topology change of the metric is considered, we must know the full gravity equation, in which case an exponential of exponential function may be obtained [12]. But, here we do not delve into an exponential of an exponential. The Euclidian action integral was found to have the form $e^{-\tilde{I}_{E}}=e^{3 \pi M_{P}^{2} / \Lambda}$. Hawking considered a scalar field in terms of $A_{\mu \nu \rho}$ (or the field strength $\left.H_{\mu \nu \rho \sigma}\right)$.

In this scenario, the quantity to calculate is the action. Even if we understand the CC in this way, we must address the following: (i) How do we assign the initial state?, (ii) How does the needed primary inflation come about in this scenario?, and (iii) How does it fit to the current DE?

With extra dimensions, there exists the no-go theorem for self-tuning solutions under some plausible conditions such that one employs the usual kinetic energy term and assumes the existence of Lorentz symmetry and 4D gravity for a large distance separation [13]. So, we try to go beyond using the standard kinetic energy (KE) term. In this regard, we note the self-tuning model by Kim, Kyae and Lee (KKL) [14]. The KKL model is worked out in the Randal-Sundrum II setup [15], with a nonstandard KE term of the field strength $H_{M N P Q}$ : $\sim 1 / H^{2}$ [14],

$$
\begin{aligned}
&-I_{E}=\int d^{5} x_{E} \sqrt{g_{(5)}}\left(\frac{1}{2} R_{(5)}-\frac{2 \cdot 4 !}{H^{2}}-\Lambda_{b}-\Lambda_{1} \delta(y)\right)=\int d y \int d^{4} x_{E} \\
&\left\{-\Psi^{4} \Lambda_{1} \delta(y)+\frac{1}{2} R \Psi^{2}+4 \Psi^{3} \Psi^{\prime \prime}+6 \Psi^{2}\left(\Psi^{\prime}\right)^{2}+\frac{2 \cdot 4 ! \Psi^{4}}{H^{2}}-\Psi^{4} \Lambda_{b}\right\} .
\end{aligned}
$$




\section{An adjustable Cosmological Constant}

The KE term with $H^{2}$ is not developing a $\mathrm{VEV}$ in the low energy limit, i.e. in the long wavelength limit $\left(\partial_{\mu} A^{\nu \rho \sigma}\right) \rightarrow 0$. So, even with $\left\langle H^{2}\right\rangle=0, H^{2}$ can be moved to the denominator, $\frac{1}{H^{2}}$, with $\left\langle H^{2}\right\rangle \neq 0$. The self-tuning solution of (1) was found in [14]. It is easy to show the existence of the nearby dS and AdS solutions also. With this self-tuning solution, we illustrate our idea of the CC solution.

\section{The wave function of the universe}

One way of doing quantum cosmology is to solve the Wheeler-DeWit equation with an appropriate boundary condition. The obtained wave function of the universe is independent of time. It is a videotape containing all the information of the universe. There are many videotapes satisfying the Wheeler-DeWit equation [7]. The probability to obtain a certain videotape is given by the wave function of the universe. If one obtains a videotape, he can run it in a film motion-picture projector with a certain definition of time to see how the videotape, containing all the fundamental constants of physics, evolves in the classical regime.

This idea of quantum cosmology must be recast with extra dimensions and branes. The KKL solution has a remarkable property as noted in [16] that the vanishing CC solution is not allowed for the parameter range of $\left|\Lambda_{1}\right| \geq \sqrt{-6 \Lambda_{b}}$, since the boundary condition at the brane $\left(\beta^{\prime} / \beta\right)_{y=0^{+}}=-\Lambda_{1} / 6$ cannot be satisfied, which is shown in the LHS figure of Fig. 2.
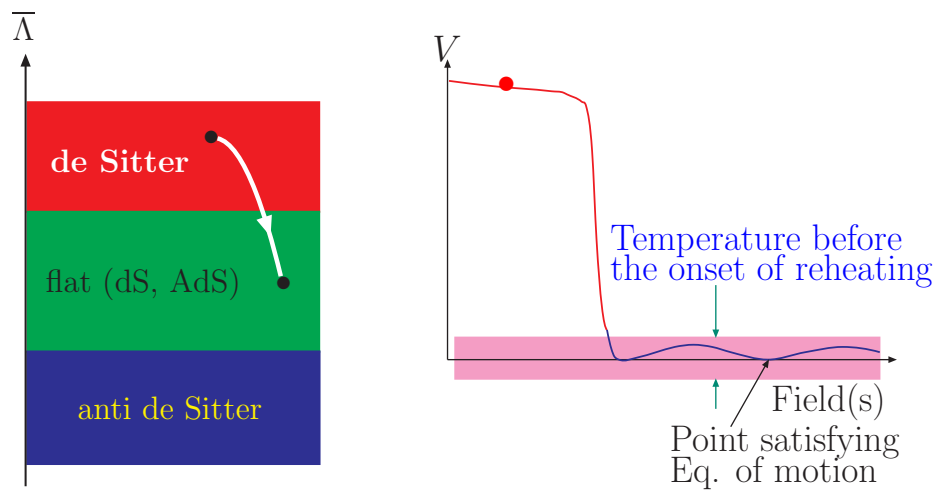

Figure 2: (a) Brane world parameters. The initial inflation takes place for $\left|\Lambda_{1}\right|>\sqrt{-6 \Lambda_{b}}$. (b) The initial inflation and the recent acceleration. In the lavender band, the CC solution is swamped and the wave function of the universe is not exactly choosing the equilibrium point.

We start with a finite range of parameter of $\Lambda_{1}$ in the top region of the LHS side figure of Fig. 2. Next, the particle physics action at the brane may change $\Lambda_{1}$ such that it falls into the central region where all the possibilities are open: flat, dS and AdS solutions. Then the inflationary period might end. This state in the central region is our initial condition.

The number of e-foldings during inflation is about 60 , or the scale factor increases by a factor $10^{26}$. So, the brane Lagrangian for inflation is tuned to satisfy this condition. Then, the initial temperature $T_{i}$ drops to $10^{-26} T_{i}$ before the inflaton reheats up the universe. At this supercooled state before reheating, we define the initial state for the probability calculation. This initial state must be fuzzy enough to allow $(0.002 \mathrm{eV})^{4}$ vacuum energy, even if our probability calculation dictates the vacuum energy should be zero. Then, we require $10^{-26} T_{i}$ be greater than 0.002 
$\mathrm{eV}$, namely $T_{i}$ should be greater than $2 \times 10^{14} \mathrm{GeV}$. Namely, we let the fuzziness of our CC solution cannot be accurate down to order $(0.002 \mathrm{eV})^{4}$, which is depicted in Fig. 2.

\section{Calculation of probability amplitude in the KKL model}

If particle physics Lagrangian does not contribute to the action integral, it is sufficient to consider the $R$ and $\Lambda$ terms only as Hawking has done. His basic argument was on the size of the Euclidian volume, suppressed by $1 / \Lambda$. The dS space volume is finite, the flat space volume is infinite, and the AdS space volume is even more infinite. If we consider the particle physics Lagrangian, as in the self-tuning model of KKL, the $1 / \bar{\Lambda}^{2}$ term contributes and the AdS volume wins [6]. This may change Hawking's view completely.

Here, I discuss what has been discussed on the $H^{2}$ Lagrangian in the Hawking type calculation, present a (probably) correct way to calculate the amplitude, and show that the KKL self-tuning model allows a finite range of parameters for which $\bar{\Lambda}=0^{-}$has the singularly large probability [6].

Hawking presented the first calculation, using $H^{2}$ term in the Lagrangian [11]. Since then, there has been discussions on which value of the $H^{2}$ term must be used in the action integral. A surface term $\epsilon^{\mu \nu \rho \sigma} H_{\mu \nu \rho \sigma}$ has been noted, which does not change the equation of motion. It turned out that it amounts to changing the sign of $H^{2}$ term in the action integral [17], from which Duff concluded that the probability amplitude for $\bar{\Lambda}=0$ is least probable with $H_{\mu \nu \rho \sigma}$. But, Wu [17] obtained the opposite result from that of Duff. This is because of the treatment on the surface term, which does not affect the equation of motion. If we follow Duff's method, it has the effect of changing the sign of $1 / H^{2}$ term inside the action integral with the surface term neglected, from $\int d^{5} x_{E} \sqrt{g_{(5)}}\left(2 \cdot 4 ! \Psi^{4} / H^{2}\right)$ to $\int d^{5} x_{E \sqrt{g_{(5)}}}\left(-2 \cdot 4 ! \Psi^{4} / H^{2}\right)$. Thus, it raises an important question, "Which method should we choose?" To our view, the confusion arises from taking a specific vacuum in their calculation [17]. As in the $\theta$-vacuum of QCD, we have the $\alpha$-vacuum of antisymmetric tensor field $H_{\mu \nu \rho \sigma}$. Duff took one extremum point corresponding to $\alpha=\pi$ and $\mathrm{Wu}$ took another vacuum corresponding to $\alpha=0$, and they obtained different results even though both satisfied equations of motion. As an illustration, we may consider $\alpha$-vacuum with the $H^{2}$ kinetic energy term. For two antisymmetric indices from $\mu, \nu, \rho$, and $\sigma$, there are six $\left({ }_{4} C_{2}=6\right)$ independent second rank antisymmetric gauge functions, for which $A_{\mu \nu \rho}$ transforms as $A_{\mu \nu \rho} \rightarrow A_{\mu \nu \rho}-\partial_{\mu} \Lambda_{\nu \rho}-\partial_{\nu} \Lambda_{\rho \mu}-\partial_{\rho} \Lambda_{\mu \nu}$; so we consider the maps of $S_{3} \rightarrow S_{3}$. Namely, there exists one type of instanton solution.

Now, we can construct a gauge invariant $\alpha$-vacuum, following the $\theta$-vacuum construction of $\mathrm{QCD},|\alpha\rangle=\sum_{n=-\infty}^{+\infty}|n\rangle e^{i n \alpha}$. In the $\alpha$-vacuum, after integrating out the $H^{2}$ field, what Duff chose is $\alpha=\pi$ and what $\mathrm{Wu}$ chose is $\alpha=0$. However, in the $\alpha$ vacuum any value of $\alpha$ is allowed, i.e. not restricted to $\alpha=0$ and $\pi$.

Now, this $\alpha$-vacuum is defined with the $1 / H^{2}$ term. We calculate the action integral for $\alpha=0$ and $\pi$ with the $1 / H^{2}$ term and for any $\alpha$ the action integral is between them. If one makes $\alpha$ a dynamical field as the QCD axion, then $\alpha$ is cosmologically settled to 0 .

Finally, we find that there exist the parameter space where $\bar{\Lambda}=0^{-}$dominates, which is the proposed CC solution. A more detailed discussion can be found in [6]. 


\section{An adjustable Cosmological Constant}

\section{Acknowledgements}

This work is supported in part by the Korea Res. Found., Grant No. KRF-2005-084-C00001.

\section{References}

[1] J. E. Kim, "Constraints on very light axion from cavity experiments," Phys. Rev. D 58, 055006 (1998); J. E. Kim and G. Carosi, "Axions and the strong CP problem," Rev. Mod. Phys. 82, 557 (2010).

[2] J. E. Kim, "Weak interaction singlet and strong CP invariance," Phys. Rev. Lett. 43, 103 (1979); M. A. Shifman, V. I. Vainstein, and V. I. Zakharov, "Can confinement ensure natural CP invariance of strong interactions?," Nucl. Phys. B 166, 4933 (1980).

[3] M. Dine, W. Fischler and M. Srednicki, "A simple solution to the strong CP problem with a harmless axion," Phys. Lett. B 108, 199 (1981); A. P. Zhitnitskii, "Possible suppression of axion-hadron interactions," Yad. Fiz. 31, 497 (1980) [Sov. J. Nucl. Phys. 31, 260 (1980)].

[4] K.-S. Choi, I.-W. Kim and Jihn E. Kim, "String compactification, QCD axion and axion-photon-photon coupling," JHEP 03, 116 (2007) [hep-ph/0612107].

[5] J. E. Kim and B. Kyae, "Flipped SU(5) from $Z_{12-I}$ orbifold with Wilson line," Nucl. Phys. B 770, 47 (2007) [hep-th/0608086].

[6] J. E. Kim, "Cosmological constant is probably adjustable in brane worlds," Phys. Rev. D 81, 123018 (2010) [arXiv:0912.2733[hep-th]].

[7] J. E. Kim, "Self-tuning of the cosmological constant," arXiv:1009.5071[hep-th].

[8] A. A. Starobinsky, "Relic gravitation radiation spectrum and initial state of the Universe," JETP Lett. 30, $682(1979)$

[9] A. H. Guth, "The inflationary Universe: A possible solution to the horizon and flatness problems," Phys. Rev. D 23, 347 (1981); A. D. Linde, "A new inflationary Universe scenario: A possible solution of the horizon, flatness, homogeneity, isotropy and primordial monopole problems," Phys. Lett. B 108, 389 (1982); A. Albrecht and P. J. Steinhardt, "Cosmology for grand unified theories with radiatively induced symmetry breaking," Phys. Rev. Lett. 48, 1220 (1982).

[10] S. Perlmutter et al. (Supernova Cosmology Project Collaboration), "Measurements of $\Omega$ and $\Lambda$ from 42 high redshift supernovae," Astrophys. J. 517, 565 (1999); A. G. Riess et al. (Supernova Search Team Collaboration), "Observational evidence from supernovae for an accelerating universe and a cosmological constant," Astron. J. 116, 1009 (1998).

[11] E. Baum, "Zero cosmological constant from minimum action," Phys. Lett. B 133, 185 (1983); S. Hawking, "The cosmological constant is probably zero," Phys. Lett. B 134, 403 (1984).

[12] S. R. Coleman, "Why there is nothing rather than something: A theory of the cosmological constant," Nucl. Phys. B 310, 643 (1988).

[13] C. Csaki, J. Erlich, C. Grojean, and T. Hollowood, "General properties of the selftuning domain wall approach to the cosmological constant problem," Nucl. Phys. B 584, 359 (2000) [hep-th/0004133].

[14] J. E. Kim, B. Kyae and H. M. Lee, "Randall-Sundrum model for selftuning the cosmological constant," Phys. Rev. Lett. 86, 4223 (2000) [hep-th/0011118]; "Selftuning solution of the cosmological constant problem with antisymmetric tensor field," Nucl. Phys. B 613, 306 (2001) [hep-th/0101027].

[15] L. Randall and R. Sundrum, "An alternative to compactification," Phys. Rev. Lett. 83, 4690 (1999) [hepth/9906064]

[16] J. E. Kim, "Inflation with blowing up solution of cosmological constant problem," JHEP 0301,042 (2003) [hep-th/0210117].

[17] M. J. Duff, "The cosmological constant is possibly zero, but the proof is probably wrong," Phys. Lett. B 226, 36 (1989); Z. C. Wu, "The cosmological constant is possibly zero, and the proof is possibly right," Phys. Lett. B 659, 891 (2008). 